\title{
Cómo medir el potencial persuasivo en Twitter: propuesta metodológica
}

\section{Miguel Moya-Sánchez ${ }^{1}$ Susana Herrera-Damas ${ }^{2}$}

Recibido: 2015-11-23

Enviado a pares: 2015-11-23
Aprobado por pares: 2015-12-14

Aceptado: 2016-01-13

DOI: 10.5294/pacla.2016.19.3.7

Para citar este artículo / to reference this article / para citar este artigo Moya Sánchez, M. y Herrera Damas S. (2016). Cómo medir el potencial persuasivo en Twitter: propuesta metodológica. Palabra Clave 19(3), 838-867. DOI: 10.5294/pacla.2016.19.3.7

\section{Resumen}

La demostrada utilidad de Twitter para una concertación colectiva alentada por nuevos prescriptores sociales y líderes de opinión ha generado numerosos estudios sobre la influencia en las redes sociales. Por lo general, la influencia es cuantificada basándose en el número de seguidores de la cuenta que se trate y en la centralidad que ocupa en su red egocéntrica de seguidores y seguidos. Sin embargo, tales estudios no están acompañados o precedidos de otros que evalúen el esfuerzo persuasivo que hace una cuenta para conseguir ser influyente. Considerada la persuasión como un acto perlocutivo utilizado tácticamente para influir, creemos conveniente contribuir al estudio de la persuasión política con un análisis del esfuerzo persuasivo de líderes y dirigentes en las redes sociales de Twitter. El método elegido para conocer el potencial persuasivo de la comunicación en Twitter ha sido el del análisis de contenido de los mensajes, que dirigentes de partidos políticos emiten en sus redes sociales egocéntricas. El presente artículo describe este método. El objetivo es determinar cuantitativamente el esfuerzo de persuasión a partir del comportamiento que denotan los mensajes en

\footnotetext{
Universidad Carlos IIII de Madrid, España.m.msz@telefonica.net

Universidad Carlos III de Madrid, España. dherrera@hum.uc3m.es
} 
Twitter. Basándonos en el uso de los mecanismos de interacción del sistema de Twitter, hemos desarrollado el concepto de índice global de potencial persuasivo (IGPP), cuyas variables vienen dadas por el número y peso comunicacional de los distintos tipos de mensajes emitidos, la frecuencia de emisión y la amplificación que encuentran en la red de seguidores.

\section{Palabras clave}

Twitter; persuasión; comunicación política; medios sociales; redes sociales (Fuente: Tesauro de la Unesco). 


\section{How to Measure Persuasive Potential on Twitter: A Methodological Proposal}

\section{Abstract}

The demonstrated usefulness of Twitter for a collective pact encouraged by new social prescribers and opinion leaders has sparked an abundance of studies on the influence that exists in social networks. Influence usually is measured according to the number of followers an account has and its importance in its egocentric network of followers and followed. However, these studies are not accompanied or preceded by others intended to evaluate the persuasive effort an account makes to become influential. With persuasion regarded as a perlocutionary act used tactically to influence, the authors consider it appropriate to contribute to the study of political persuasion with an analysis of the persuasive efforts of leaders and managers in Twitter's social networks. An examination of the contents of messages political party leaders issue within their egocentric social networks was the method selected to identify the persuasive potential of communication on Twitter. The article describes this method. The objective is to measure persuasive effort quantitatively, based on the behavior implied by messages on Twitter. Using the mechanisms for interaction in the Twitter system, the authors have developed the notion of a global index of persuasive potential (GIPP), the variables of which are found in the number and communicative weight of the various types of messages emitted, the frequency of emission and the amplification found in the network of followers.

\section{Keywords}

Twitter; persuasion; political communication; social media; social networks (Source: Unesco Thesaurus). 


\section{Como medir o potencial persuasivo no Twitter: proposta metodológica}

\section{Resumo}

A demonstrada utilidade do Twitter para uma coalizão coletiva encorajada por novos decisores sociais e líderes de opinião tem gerado numerosos estudos sobre a influência nas redes sociais. Em geral, a influência é quantificada baseando-se no número de seguidores da conta em questão e na centralidade que ocupa em sua rede egocêntrica de seguidores e seguidos. Contudo, tais estudos não estão acompanhados ou precedidos de outros que avaliem o esforço persuasivo que uma conta faz para conseguir ser influente. Considerada a persuasão como um ato perlocutório utilizado taticamente para influenciar, acredita-se conveniente contribuir para o estudo da persuasão política com uma análise do esforço persuasivo de líderes e dirigentes nas redes sociais do Twitter. $\mathrm{O}$ método escolhido para conhecer o potencial persuasivo da comunicação no Twitter tem sido a análise de conteúdo das mensagens que líderes de partidos políticos emitem em suas redes sociais egocêntricas. $\mathrm{O}$ presente artigo descreve esse método. $\mathrm{O}$ objetivo é determinar quantitativamente o esforço de persuasão a partir do comportamento que as mensagens no Twitter denotam. Baseando-se no uso dos mecanismos de interação do sistema do Twitter, desenvolveu-se o conceito de índice global de potencial persuasivo (IGPP), cujas variáveis são dadas pelo número e pelo peso comunicacional dos diferentes tipos de mensagens emitidas, a frequência de emissão e a amplificação que encontram na rede de seguidores.

\section{Palavras-chave}

Twitter; persuasão; comunicação política; meios sociais; redes sociais (Fonte: Tesauro da Unesco). 


\section{Introducción}

La relevancia de Twitter como instrumento comunicacional para el activismo social y político se evidencia con el uso que las administraciones públicas, los gobernantes y los líderes de opinión hacen del medio (McNair, 2011).Su uso como instrumento de movilización es recurrente en escenarios de control político gubernamental (Gaffney, 2010; Lotan et al., 2011). En situaciones de crisis, Twitter se ha revelado como un medio eficaz para la concertación colectiva por parte de activistas que no gozaban de la posibilidad de convocatoria que suelen tener los partidos en el Gobierno en los medios de comunicación de masas. Nos referimos a casos ejemplarizantes, como el de las protestas de los reformistas iraníes en 2009 (Zhou et al., 2010) o el de las revueltas populares que dieron lugar a la conocida como Primavera Árabe (Hopcroft, Lou y Tang, 2011; Khondker, 2011).

No obstante, su empleo no se ha limitado a situaciones excepcionales, sino que también ha sido normalizado como medio para la comunicación política por miembros de o candidatos a parlamentos nacionales de América Latina, España, el Reino Unido, Australia o los Estados Unidos . Son numerosos los dirigentes políticos que lo utilizan para una mayor transparencia y divulgar sus agendas públicas en Twitter (Aharony, 2012). Por su parte, los ciudadanos comunes de sociedades con regímenes democráticos han hecho también de las redes sociales de Twitter un ágora contemporánea, donde se airean las diferencias políticas y se debate de modo informal (Tumasjan et al., 2010; Golbeck, Grimes y Rogers, 2010; Lassen y Brown, 2011; Larsson y Moe, 2012; Park, 2013).

Asimismo, Twitter es un espacio donde se difunde la frustración ciudadana, en especial de los más jóvenes, y a veces de los más instruidos, ante las políticas que las instituciones de la democracia representativa han aplicado para afrontar la crisis económica que se inició en 2008. Algunos de los casos más representativos de movimientos sociales que han aglutinado el malestar social con una utilización intensiva de los medios sociales son el de los indignados en España, el de los jóvenes de México (\#YoSoy132 en México) o los de Chile (la revolución de los pingüinos), Occupy Wall Street en los Estados Unidos y Aganaktismenoi en Grecia. En general, estos movimientos cuestionan los canales tradicionales de parti- 
cipación ciudadana en el debate político y la representatividad de los dirigentes políticos (Parmelee y Bichard, 2011). Sin embargo, casos como el de los jóvenes chilenos demuestran que una mayor frecuencia en el uso de medios sociales se correlaciona con una relación positiva en la participación cívica (Arriagada y Schuster, 2008; Scherman y Arriagada, 2010).

Los procesos de información, discusión o concertación con fines políticos que se desarrollan en las redes sociales se caracterizan por una relación de comunicación directa, interactiva y en tiempo real entre dirigentes o activistas sociales devenidos líderes de opinión y ciudadanos comunes. De este modo, el medio social contribuye a un diálogo político entre dirigentes y dirigidos, gobernantes y gobernados, en el que el ciudadano común tiene la oportunidad de interpelar al político, responderle o informarle, sin la intermediación y el encuadre propio de los medios de comunicación tradicionales.

En esa comunicación, que se lleva a cabo con fines políticos, tiene lugar un proceso persuasivo, que incluye la propaganda, con la intención final de influir: ${ }^{3}$ la influencia como finalidad, la persuasión como táctica para conseguir ser influyente. Ambas son inherentes a la comunicación política (Johnson-Cartee y Copeland, 2003; Yanes, 2007; Bennett y Iyengar, 2008), y se ha verificado su permanencia en las redes sociales, donde los políticos emplean estrategias de persuasión y seducción similares a las que se pueden observar en otros procesos comunicativos habituales. No obstante, en Twitter, la exposición tiene un mayor rigor de formalidad para no incurrir en riesgos por errores tácticos que perjudiquen la imagen del político (Mancera y Pano, 2013). La utilización de Twitter como medio para la comunicación social y su aprovechamiento político, tanto en circunstancias de normalidad democrática como en revueltas populares, ha despertado el interés académico por conocer cómo se manifiesta la influencia en unas redes de comunicación horizontal y masiva que favorecen la autocomunicación de masas (Castells, 2008).

3 Según la RAE, persuadir es "inducir, mover, obligar a alguien con razones a creer o hacer algo". Influir es "ejercer predominio, o fuerza moral”. Una comunicación política persuasiva sería aquella que dirigentes o activistas políticos llevasen a cabo con el propósito de inducir a sus seguidores a adoptar actitudes que favorezcan intereses políticos del partido o movimiento que aquellos representan. No evaluamos el resultado del esfuerzo persuasivo que requeriría analizar los cambios en la conducta de los receptores que se hubieran producido como consecuencia de los mensajes del emisor. 
Los estudios relacionados se han encaminado, con preferencia, a demostrar teóricamente que un determinado grado de centralidad en la red social donde se produce el intercambio de mensajes lleva asociado un correlativo nivel de influencia, de modo que los comunicantes con un elevado número de seguidores son influyentes (Hanneman, 2001). La metodología aplicada ha sido la del análisis de redes mediante la teoría de grafos (Molina, 2001; Requena, 2003; Trusov, Bodapati y Bucklin, 2009; Cha et al., 2010; Weng et al., 2010; Bakshy et al., 2011a; Bakshy et al., 2011b; Anger y Kittl, 2011; Parmelee y Bichard, 2011; Aral y Walker, 2012). En algunos casos, además del tamaño de la red del comunicante, también se ha tenido en cuenta el número de tuiteos emitidos y las menciones recibidas (Cha et al., 2010). Otras veces se ha incorporado como un factor relevante el grado de afecto a la cuenta que revelan los seguidores en sus mensajes (Stieglitz, Brockmann y Xuan, 2012).

Sin embargo, estos estudios no han aplicado técnicas metodológicas enraizadas en la tradición investigadora de la influencia a través de la comunicación personal (Katz y Lazarsfeld, 1955; Merton, 1948), como es la que tiene lugar en las redes sociales de Twitter. Tal vez el elevado coste de contrastar la influencia que los mensajes en Twitter han tenido en la configuración de algunas de las actitudes u opiniones de los receptores haya resultado disuasorio a la hora de aplicar metodologías tradicionales en el estudio de la influencia personal, lo cual es lo que ha motivado que optemos por evaluar el esfuerzo persuasivo del emisor antes que los efectos de sus mensajes en sus seguidores.

Nos alineamos, pues, metodológicamente, con otras líneas de investigación sobre el mismo tema, aunque limitadas al estudio de la presencia y actividad en las redes sociales de dirigentes políticos (Álvarez y Rodríguez, 2014) o de Twitter como un medio para la comunicación política (Abejón, Sastre y Linares, 2012; Borondo et al., 2012; García, García y Varona, 2012; Izquierdo, 2012). Centramos nuestra investigación en el análisis del comportamiento del dirigente político en su desempeño como comunicante emisor, para determinar su potencial persuasivo mediante el estudio de la tipología y frecuencia de sus mensajes. Para ello, hemos aplicado un método efectivo y tradicional en la investigación social, como es el análisis de 
contenido (Berelson, 1971; Krippendorff, 1989; Krippendorff y Wolfson, 1990) aplicado al significante del mensaje, a su código, "revelador de realidades subyacentes" (Bardin, 1996, p. 104).

\section{Persuasión comunicacional en Twitter}

Dada por cierta la relevancia de la comunicación personal para la influencia (Katz y Lazarsfeld, 1955), los procesos persuasivos subyacentes a los mensajes que los dirigentes políticos emiten en Twitter $-\mathrm{y}$ que reciben sin intermediación - se revelan claves para el éxito de una táctica orientada a conseguir el apoyo a un determinado programa o acción política. En ese medio, el dirigente político tiene la oportunidad de desplegar sus habilidades persuasivas, reforzando su presencia en los tradicionales a la vez que presenta una imagen pública personalizada y diferenciada de la colectiva del partido al que pertenece. Una comunicación persuasiva tiene éxito si sus receptores cambian su actitud política, bien mediante un procesamiento sistemático que tiene en cuenta el contenido del mensaje, bien heurísticamente, considerando facetas del comunicador. En el primero de los casos, el receptor atiende las implicaciones de los argumentos presentados, lo cual requiere motivación y habilidad para procesar el mensaje a fondo. Cuando estas son insuficientes o el mensaje presenta un déficit de contenido, el prestigio del comunicador, su amabilidad o el afecto que genera son los elementos que, en el proceso persuasivo, sustituyen a los argumentos (Schwarz, Bless y Bohner, 1991; Chaiken, Wood y Eagly, 1996; Chaiken y Maheswaran, 1994).

Las características de la comunicación en Twitter —intercambio de mensajes de hipertexto ${ }^{4}$ de extensión limitada a 140 caracteres - facilitan que el procesamiento que predomine entre los receptores sea el heurístico. A ello debe coadyuvar que el mensaje proceda de un dirigente político que ostente poder o, al menos, se le atribuya un estatus social superior al del ciudadano común. Por tanto, en el impacto persuasivo de la comunicación en Twitter, el comportamiento comunicacional del dirigente, es decir, su dis-

4 El texto puede contener caracteres, como RT o @, que son metadatos — que indican, respectivamente, mensaje retransmitido o dirección de otro comunicante-, o hiperenlaces a otras direcciones de internet o mensajes de Twitter. Estas posibilidades dan categoría hipertextual al contenido de los tuiteos. 
posición a interesarse por las opiniones de otros comunicantes desempeña un papel de relevancia, lo cual se puede hacer de distintas maneras; por ejemplo, invocando su participación mediante la mención expresa, participando en el debate - con respuestas a otros mensajes - o con el empoderamiento de otros ciudadanos que se produce al darle la oportunidad de ampliar su audiencia y refrendar sus opiniones con los retuiteos. La persuasión a la que aquí nos referimos es la vinculada al emisor; en concreto, al esfuerzo que hace este por inducir en el receptor un cambio de actitud en una dirección determinada. ${ }^{5}$ La persuasión ejercida con una presencia activa en Twitter ha sido adoptada como táctica de comunicación por dirigentes de los partidos políticos tradicionales que se han incorporado a las redes sociales tras el éxito alcanzado en ellas por nuevos líderes de opinión; por ejemplo, en España, los vinculados al movimiento del 15M, y similares.

La adopción de nuevas formas de comunicación política que se desarrollan en Twitter encuentra fundamento en la teoría de la difusión de la innovación (Rogers, 1983; Chang, 2010). Tras constatar que la presencia de los dirigentes políticos españoles se ha normalizado en Twitter (Moya, 2015), procede analizar el potencial persuasivo que se deriva de sus mensajes en ese medio. Su cuantificación hace que sea posible establecer comparaciones objetivas de unas cuentas con otras. Ello ha requerido elaborar un constructo que nos permita medir la persuasión a partir de los componentes del sistema comunicacional de Twitter que son operables aritméticamente. El número de tuiteos de cada tipo emitidos por una cuenta, su peso comunicacional, la frecuencia de emisión y el grado de propagación que consiguen son los componentes (variables independientes) que nos permiten determinar cuantitativamente el valor del constructo o variable dependiente. Llamamos a este índice global del potencial persuasivo de una cuenta (IGPP). Dado que deseamos medir el potencial persuasivo con respecto a una materia —la política-, el conjunto de mensajes por estudiar debe ser monotemático y homogéneo, para que pueda ser utilizado comparativamente.

5 El proceso persuasivo en Twitter se caracteriza por una comunicación centrada en la venta de determinadas políticas o de una imagen en particular (propaganda), que se lleva a iniciativa de uno mediante mensajes que llegan a muchos, que no son los elegidos por el emisor. Esa comunicación es bidireccional y no controlada por el emisor ni por el medio que la vehicula, lo cual anima al ciudadano común. El anonimato rompe la barrera que supone la espiral de silencio entre quienes tienen opiniones minoritarias (Noëlle-Neumann, 1995). 
En nuestro caso, tal conjunto está constituido por aquellos mensajes con un contenido político. Así, y en general, será posible comparar el potencial persuasivo de un analista político con el de un periodista, un dirigente político u otro líder de opinión, y entre miembros de cada uno de estos grupos. En un mismo colectivo, el IGPP sitúa a cada cuenta en su percentil correspondiente, lo cual facilita establecer perfiles de referencia - los de mayor IGPP_, así como identificar acciones de mejora para las cuentas que presenten un IGPP más bajo. En este análisis, un factor básico para determinar el comportamiento persuasivo de una cuenta es la frecuencia con la que esta genera mensajes comunicacionales.

Los mensajes que incluyan menciones personales o que sean respuestas a otros denotan un comportamiento comunicacional superior a aquellos otros mensajes que no aludan a nadie. Por otra parte, la retransmisión en cascada de un mensaje — el retuiteo - aumenta de un modo impredecible su área de difusión y, por tanto, el número de potenciales receptores. Como consecuencia, el efecto persuasivo del mensaje retuiteado es susceptible de verse sensiblemente aumentado con respecto al que hubiera alcanzado si no hubiese sido retransmitido. En suma, los mensajes que son retuiteados o que respondan o aludan a otra cuenta personal tienen un peso comunicacional superior al de los tuiteos básicos, que son aquellos que no aluden a nadie, ni son respuesta ni tampoco son retransmitidos. Con esta lógica, establecemos que, según su potencial comunicador, los mensajes en Twitter pueden ser de los siguientes tipos:

- Tuiteos básicos $(\mathrm{Tb})$. Son mensajes generados por la cuenta que no mencionan ni responden a otra cuenta.

- $\quad$ Respuestas (Rp). Son mensajes emitidos como respuesta a otros tuiteos.

- Menciones (Me). Se trata de alusiones nominales a otras cuentas.

- Retuiteos (Rtn). Son los mensajes no generados sino recibidos y retransmitidos por la cuenta.

Para calcular el potencial persuasivo, utilizaremos las siguientes dimensiones:

- $\quad$ Actividad de la cuenta, calculada a partir del número medio diario de tuiteos emitidos. La medimos con el que llamamos coeficiente de actividad (Cact). 
- Actividad generadora de comunicación. Tiene en cuenta acciones conversacionales — respuestas y menciones - o que favorecen la comunicación — seguimiento recíproco- Viene determinada por el coeficiente de comunicación $(\mathrm{Ccm})$ que describimos más adelante.

- Penetración de los mensajes emitidos, que medimos mediante otro coeficiente, el de alcance de la cuenta (Alc).

\section{Coeficiente de actividad}

La actividad total de una cuenta (Act) es el número de mensajes de cualquier tipo (Msg) que ha emitido esa cuenta durante un periodo (Per). Incluye los tuiteos básicos generados por la cuenta $(\mathrm{Tb})$, los que son respuestas a los emitidos por otras cuentas, en general, aquellas a las que sigue ( $R p)$ y las retransmisiones que la cuenta ha hecho de mensajes creados por otras (Rtn). Matemáticamente, la expresamos así: $A c t=(T b+R p+R t n)$. Esta fórmula toma valores absolutos acumulados a lo largo del tiempo, lo que no permite establecer comparaciones.

Para dilucidar si una cuenta es o no más activa que otra, debemos tener en cuenta el periodo en el cual fueron emitidos esos mensajes (Per). ${ }^{6}$ De ahí surge el concepto de 'coeficiente de actividad' (Cact), que nos indica la actividad media de una cuenta en un periodo que es igual para todas las cuentas. Para calcular el coeficiente Cact, hemos tomado el día como unidad de tiempo. Por tanto, formulamos así el coeficiente de actividad: Cact $=(\mathrm{Tb}+\mathrm{Rp}+\mathrm{Rtn}) /$ Per.

\section{Coeficiente de comunicación}

Llamamos coeficiente de comunicación $(\mathrm{Ccm})$ al esfuerzo que hace una cuenta para generar conversación. En nuestra propuesta metodológica, el Ccm depende de la relación de seguimiento que tenga con otras cuentas y de la comunicación propiamente dicha que sostenga con ellas. Intuitivamente, se tiende a asignar un mayor efecto comunicacional a una cuenta cuanto más elevado sea el número de sus seguidores. Sin embargo, es necesario tener en cuenta dos factores cualitativos: uno es el tipo de esos segui-

6 Número de días transcurridos desde que se emitió el primer mensaje hasta la fecha de recogida de datos. 
dores — si son únicos o recíprocos- El otro es el significado que denota el tipo de mensaje. Es decir, tanto la relación de seguimiento de una cuenta con sus seguidores como la relación de comunicación que mantenga con ellos son componentes de su acción comunicadora. La relación de seguimiento es la que los usuarios de Twitter establecen en su red egocéntrica y que no presupone necesariamente ningún intercambio de mensajes. La relación de comunicación es la que se establece, tácitamente, cada vez que se retransmite un mensaje generado por otra cuenta o, expresamente, cuando se alude o responde a alguien. La relación de seguimiento denota interés a priori, por lo que puedan decir las cuentas seguidas. La relación de comunicación manifiesta interés por lo dicho por otro o por conversar; evidencia una realidad.

\section{Relación de seguimiento y coeficiente de seguimiento (Cfs)}

La relación de seguimiento entre dos cuentas está asociada al flujo de mensajes que pueda existir entre ambas. En Twitter, tiene dos manifestaciones: una unidireccional, que es la del seguidor que no es correspondido por el seguido, y otra bidireccional o recíproca, que es aquella que se produce cuando el usuario Ui es seguidor de Uj, y este, a su vez, sigue a Ui. Las causas para la formación de relaciones de seguimiento recíproco que tienen lugar en las redes sociales de Twitter se entienden desde la teoría social del equilibrio, que establece que, en las relaciones interpersonales, una situación equilibrada es aquella en la que los individuos adoptan una misma actitud respecto del otro (Taylor, 1967; Heider, 1982; Khanafiah y Situngkir, 2004). Por el contrario, las situaciones no equilibradas se caracterizan por que los miembros de una díada tienen opiniones diferentes el uno del otro, lo cual puede ser debido a una falta de homogeneidad personal o a una escasa familiaridad. A diferencia de las situaciones equilibradas, en las no equilibradas los participantes no mantienen una relación de criterio u opinión común. Podemos establecer un paralelismo entre el tipo de relación de seguimiento en Twitter motivado por razones políticas y el de relación interpersonal. Aunque Twitter muestra un nivel de reciprocidad de tan solo $22.1 \%$ (Kwak et al., 2010), entendemos que los líderes políticos incorporan a Twitter su popularidad fuera de línea, de modo que entre sus 
seguidores abundan los afines más que los contrarios políticos (Weng et al., 2010), asíla homofilia política no implique, necesariamente, una influencia mayor que los lazos sociales (Cha et al., 2010). El seguimiento recíproco que tiene lugar cuando el dirigente político se convierte en seguidor de alguno de sus seguidores es una relación bidireccional. Posiblemente se trata de una relación motivada por la afinidad de quienes comparten los mismos valores políticos que presenta un equilibrio comunicacional.

Por su parte, la relación unidireccional en una díada no presenta con igual fortaleza la comunicación que pueda existir entre sus miembros. Se trata de una relación de comunicación no equilibrada, más probablemente la de una díada con una afinidad política más atenuada o inexistente. Este equilibrio comunicativo es instrumental para analizar la sentiment relations, pues, cuando la relación de comunicación evoluciona hacia un estado de equilibrio, fortalece la interacción social y la solidaridad en las redes sociales interpersonales (Khanafiah y Situngkir, 2004). ${ }^{7}$ Por tanto, establecemos que el potencial de interacción debe ser mayor en una relación de seguimiento recíproco que en la de una relación unidireccional: la reciprocidad en el seguimiento asegura que entre los miembros de una díada de usuarios fluye un mayor número de mensajes. Procede, por tanto, asignar un valor a la relación recíproca superior al de la unidireccional. Para determinarlo, razonablemente, hemos tenido en cuenta el número de relaciones unidireccionales que equivalen a una relación recíproca, como sigue: la relación de seguimiento entre dos usuarios, Ui y Uk, vale 0 cuando ninguno de ellos es seguidor del otro. A la relación de seguimiento $\mathrm{Ui} \rightarrow \mathrm{Uj}$ (o $\mathrm{Uj} \rightarrow \mathrm{Ui}$ ), le asignamos el valor de 1 . El problema que se nos plantea es cuánto mayor es la relación recíproca, $\mathrm{Ui} \leftrightarrow \mathrm{Uj}$, con respecto a una relación unidireccional del tipo $U i \rightarrow U j$ (o $U j \rightarrow U i)$. Veamos cuál puede ser ese valor con la ayuda de la figura 1 :

7 A la reciprocidad en las redes sociales no es ajeno el fenómeno de homofilia (Rogers y Bhowmik, 1970; Wu, Hofman, Mason y Watts, 2011; McPherson, Smith-Lovin, y Cook, 2001) que se manifiesta como una mayor probabilidad de relación entre personas que comparten gustos o ideas. Ello explica que las redes personales sean más homogéneas en aspectos sociodemográficos, comportamientos, ideas o características personales (McPherson et al., 2001; Weng, Lim, Jiang y He, 2010). 


\section{Figura 1. Escenarios básicos de relación de seguimiento en Twitter}

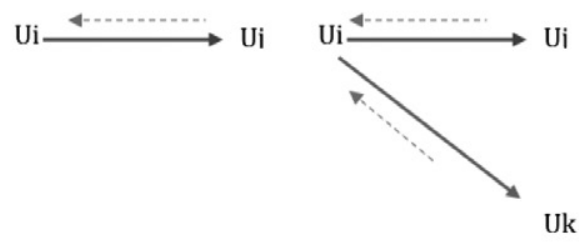

Escenario s1

Escenario s2

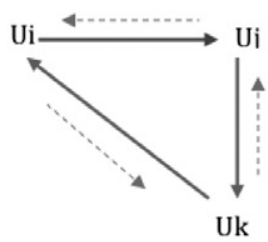

Escenario $\mathrm{s} 3$

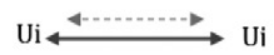

Escenario 54

NOTA:

Relación de seguimiento

Relación de comunicación (dirección de los mensajes)

Fuente: elaboración propia.

En el escenario s1, Ui tiene una única relación de seguimiento, la que le permite recibir lo que emita $\mathrm{Uj}$, y dos en el s2, con $\mathrm{Uj}$ y Uk. En ninguno de esos casos, Uj tiene acceso a lo que emita con Ui, por tanto, no hay comunicación entre ellos a partir de lo que exprese Ui. En el escenario s3, Uj tiene la posibilidad de conocer lo que emita Ui si esto es retuiteado por Uk. Por consiguiente, la posibilidad de Uj para conocer lo que diga Ui requiere, como premisa, que exista una relación de seguimiento unidireccional entre cada una de las tres cuentas. Alternativo al escenario s3 es el s4, en el que la relación recíproca $U i \leftrightarrow U j$ tiene, como mínimo, el mismo valor comunicacional que el que sea posible alcanzar con las relaciones existentes en s3. Así, en s4 todo lo que emita Ui puede ser leído por Uj. Indudablemente el escenario 33 es menos eficaz que el s4, desde la perspectiva de la comunicación, aunque ambos usuarios pueden leer lo que emita el otro siempre que Uk actúe como retransmisor. Dado que, para que exista comunicación bidireccional entre $\mathrm{Ui} \mathrm{y} \mathrm{Uj}$ en $\mathrm{s} 3$, se requieren tres relaciones de seguimiento unidireccional, y puesto que, para el mismo propósito, en s4 solo se requiere la de seguimiento recíproco, asignamos a este un valor comunicacional tres veces superior al de la relación unidireccional.

Obviamente, en s3 no se asegura una comunicación bidireccional entre ambos usuarios, aunque sí se hace posible. En consecuencia, podemos decir que la relación de seguimiento recíproco tiene un valor comunicacional, como mínimo, el triple del que tiene la relación unidireccional. Por 
tanto, hacemos de la relación de seguimiento un factor multiplicador de la capacidad comunicadora de los mensajes, pues, cuanto mayor sea el número de seguidores recíprocos de una cuenta, mayor será la probabilidad de interactuar con ellos. A la vez, el seguimiento recíproco producirá un efecto de retroalimentación comunicacional, ya que, con un aumento del número de seguidores recíprocos, se incrementarán también las respuestas y los retuiteos (Hopcroft, Lou y Tang, 2011). Por otra parte, si Ui y Uj se conocen a través de retuiteos de seguidores comunes, aumentará la probabilidad de que establezcan entre sí un enlace recíproco. Así, las cuentas con un mismo número de seguidores tendrán un potencial de interacción mayor cuanto mayor sea el número de seguidores recíprocos. De este modo, relativizamos la importancia del número de seguidores únicos. Lo expresamos matemáticamente con la siguiente fórmula: $\mathrm{Cfs}=($ Seg-u $+3 \times$ Seg-r $) /$ Tseg, donde Seg-u es el número de seguidores únicos (siguen una cuenta pero no son seguidos por ella), Seg-r es el de seguidores recíprocos (siguen una cuenta y son seguidos por ella) y Tseg es la suma de ambos, el número total de seguidores. El valor de Cfs oscilará entre 1 su mínimo cuando Seg-r = 0, y 3, el máximo, cuando Seg-r = Tseg. Un ejemplo que ilustra la importancia que atribuimos a los seguidores recíprocos es el de $\mathrm{Ui}$ y $\mathrm{Uj}$, dos usuarios con 120 y 200 seguidores, respectivamente; Ui tiene 30 seguidores recíprocos; y Uj, 10. Sus respectivos Cfs son 1.5 y 1.1. La cuenta Ui presenta, inicialmente, un perfil más comunicacional que el de $\mathrm{Uj}$, debido al mayor número de seguidores recíprocos. De ello se deriva una mayor interacción con sus seguidores que la que tiene Uj y, por tanto, de más oportunidades de ser más persuasiva que esta. El número de seguidores representa la popularidad de la cuenta, pero, por sí solo, no es factor único determinante de los efectos de la comunicación, tal como han concluido Cha et al. (2010).

\section{Relación de comunicación y coeficiente de comunicación (Ccm)}

La relación de comunicación de dos cuentas se materializa cuando un mensaje de una de ellas es respondido o retuiteado por la otra o la menciona. Así, un tuiteo generado $(\mathrm{Tg})$ por Ui y respondido por $\mathrm{Uj}$ forma parte de una conversación entre ambos. También el retuiteo que Uj pueda hacer de un mensaje de Ui establece una relación de comunicación entre $\mathrm{Ui} \mathrm{y} \mathrm{Uj,} \mathrm{aun-}$ 
que en este caso es indirecta. Desde la perspectiva de la persuasión, las respuestas y los retuiteos tienen un valor comunicacional superior al del tuiteo básico. Con este, el dirigente político difunde información u opinión o propaganda política. Informa más que conversa o comunica. En cambio, la respuesta que haga es un acto conversacional.

De un modo similar, la mención que expresa a una cuenta en el texto de un mensaje tiene una dimensión conversacional que no existe en el tuiteo básico: los mencionados pueden interpretar que la alusión personal y explícita es una invitación a expresar su opinión; en suma, a responder, a participar en la conversación en línea.

A los mensajes retuiteados, les atribuimos también un peso comunicacional superior al que tendrían de no haber sido retransmitidos. Lógicamente, las retransmisiones que se hacen de un determinado tuiteo, al tener, como consecuencia inmediata, un mayor número de receptores, hacen que el mensaje que se retransmite tenga un mayor potencial persuasivo. El retuiteo en cascada amplía la audiencia inicial que viene determinada por el tamaño de la red egocéntrica de la cuenta que difunde el mensaje.

$\mathrm{Al}$ incremento de audiencia y, por tanto, de potenciales persuadidos que causa esta ampliación del área de difusión, hay que añadir otras probables consecuencias, como son la promoción y potencial mejora de la imagen pública del autor del tuiteo, lo cual, a su vez, puede derivar en un aumento del número de sus seguidores, así como en el de respuestas al mensaje. Para cuantificar el peso comunicacional de los diferentes tipos de mensajes que fluyen de una cuenta, partiremos de que el tuiteo básico tiene un carácter netamente informativo, por lo que le asignamos un peso comunicacional nulo. Esta consideración se fundamenta en un modelo de comunicación que incluye el retorno — feedback — como parte del proceso (Schramm, 1954). Puesto que el tuiteo básico carece de feedback directo y explícito - es un tuiteo que ni menciona ni responde a nadie ni es generador de ulteriores respuestas posibles mediante el retuiteo-, su peso comunicacional debe ser cero. Por el contrario, la respuesta es el paradigma de la conversación. En un caso hipotético cuando solo se emitieran tuiteos 
básicos y respuestas, aquellos representarían un comportamiento informativo, mientras que estas denotarían el comunicativo.

Con un criterio lógico, si asignamos un peso comunicacional nulo al tuiteo básico, el de la respuesta podría ser cualquiera superior a cero. Adoptamos el peso 1 para los tuiteos que son respuestas. La determinación del peso comunicacional del retuiteo se apoya en el hallazgo de Hopcroft, Lou y Tang (2011), que determina que el retuiteo tiene una contribución a la relación recíproca del orden de $15 \%$, mientras que la respuesta lo hace con $9 \%$. Es decir, el retuiteo contribuye $66 \%$ más que la respuesta al incremento del número de seguidores recíprocos. Como el seguimiento recíproco favorece la comunicación, deberíamos, en línea con las conclusiones del estudio referido, dotar al retuiteo de un peso comunicacional superior al de la respuesta en $66 \%$. En consecuencia, establecemos que el retuiteo tiene el peso comunicacional de 1.66 respuestas. La mención también tiene un cierto peso comunicacional, ya que invoca a otro comunicante de manera explícita. Denota una intención de comunicar, es manifiestamente proactiva, mientras que en la respuesta esa intención es reactiva. Dado que a lo que damos valor comunicacional es al esfuerzo por ser persuasivo, entendemos que la mención es más explícitamente invocadora, y por consiguiente denota un valor comunicacional añadido. ¿Qué peso procede asignarle en relación con la respuesta? Lógicamente debe estar comprendido entre el de esta, 1 , y el del tipo de mensaje que genera más comunicación, el del retuiteo, 1.66 (Hopcroft, Lou y Tang, 2011).

Al no disponer de referencias de investigación al respecto, convenimos en relativizarlo y asignarle el valor medio que encontramos entre la respuesta y el retuiteo; es decir $(1.66+1) / 2=1.33$. Por tanto, una mención equivale a 1.33 respuestas. Una vez cuantificados los pesos comunicacionales de estas tres variables básicas - la respuesta, la mención y el retuiteo-, disponemos, inicialmente, de la siguiente expresión matemática para el esfuerzo comunicador: $(\mathrm{Rp}+1.33 \times \mathrm{Me}+1.66 \times \mathrm{Rtp})$, donde $\mathrm{Rp}$ y Me son, respectivamente, las respuestas y las menciones realizadas por la cuenta, $y$ Rtp es el número de retransmisiones (retuiteos) que los seguidores de esa cuenta han hecho de los tuiteos emitidos por esta. 
Para que el concepto de 'esfuerzo comunicador' pueda ser comparable, debe ser relativizado con respecto a la actividad emisora desarrollada $($ Act $=\mathrm{Tb}+\mathrm{Rp}+\mathrm{Rtn})$. Es decir, debe ser medido con respecto a la producción total de mensajes propios emitidos (tuiteos, retuiteos y respuestas). Así obtenemos un valor más preciso y operativo del esfuerzo comunicador para un determinado número de mensajes emitidos: $(\mathrm{Rp}+1.33 \times \mathrm{Me}+1.66 \times \mathrm{Rtp}) /(\mathrm{Tb}+\mathrm{Rp}+\mathrm{Rtn})$. Sin embargo, esta expresión matemática del esfuerzo para comunicarse no tiene en cuenta las relaciones de seguimiento, de cuyo estudio hemos deducido que, a un mayor grado de reciprocidad en el seguimiento, corresponde también una mayor interacción potencial. Por tanto, la formulación final del coeficiente de comunicación deberá ser modulada por la del coeficiente de seguimiento. Lo que expresamos con la siguiente fórmula: $\mathrm{Ccm}=\mathrm{Cfs} \times((\mathrm{Rp}+1.33 \times$ $\mathrm{Me}+1.66 \times \mathrm{Rtp}) /(\mathrm{Tb}+\mathrm{Rp}+\mathrm{Rtn}))$.

El coeficiente de comunicación es un indicador de la intencionalidad conversacional de la cuenta, del esfuerzo o habilidad para establecer conversaciones. Del Ccm de un dirigente podremos deducir si su presencia en Twitter sigue la inercia de la comunicación unidireccional habitual de los medios tradicionales — donde predomina el mensaje informativo- o si, por el contrario, su comportamiento en las redes sociales es más conversacional, lo cual nos permitirá establecer la frontera entre cuentas informativas de un determinado colectivo homogéneo. Para un mismo colectivo, diremos que son informativas las cuentas con un Ccm inferior a la media, mientras que a las que las que superen esa frontera las calificaremos como cuentas comunicativas.

\section{Alcance (Alc)}

Hemos aplicado a la persuasión la conclusión de Cha et al. (2010) de que el número de seguidores tiene un peso relativo en la influencia de una cuenta. Así, el efecto persuasivo no lo hacemos depender únicamente del número de seguidores. Ateniéndonos a la lógica, deducimos que el número de retransmisiones sí es un indicador que nos aproxima, más y mejor que el número de seguidores, a una determinación del alcance de los mensajes. Precisamente porque el retuiteo amplía la dimensión inicial del campo de persuasión que constituye la red personal (Bakshy et al., 2011a). Dado que 
el proceso de difusión es en cascada — originado por los seguidores inmediatos, de estos a sus seguidores, y así sucesivamente-, computar el número de retransmisiones que ha conocido un mensaje nos permite hacer un cálculo exacto del alcance mínimo que este ha tenido (como mínimo, han habido tantos seguidores que han leído el mensaje como retuiteos).

En lugar de especular con el número de seguidores, recurrimos a un valor estadístico, como es la propagación que han tenido los mensajes retuiteados. Llamamos coeficiente de propagación o alcance (Alc) de una cuenta al número medio de retransmisiones que han tenido sus tuiteos. Matemáticamente es el cociente entre el número total de retransmisiones de los mensajes emitidos por una cuenta (txMsg) y el número de estos (Rtt), es decir, $\mathrm{Alc}=\mathrm{txMsg} / \mathrm{Rtt}$. De dos cuentas a las que les hayan retuiteado el mismo número de mensajes, diremos que tiene mayor alcance aquella que haya tenido un mayor número de retransmisiones de tuiteos emitidos por ella. Esto merece las siguientes consideraciones:

- Habrá más retransmisiones de los tuiteos más valorados, que probablemente serán los más influyentes.

- El número de retransmisiones indica el número mínimo de lectores del tuiteo retransmitido. Es un número más significativo que el de seguidores que son solo lectores potenciales, pues el momento de emisión no necesariamente coincide con el de máximo número de seguidores conectados.

\section{Índice global de potencial persuasivo (IGPP)}

El IGPP es un indicador del esfuerzo de comunicación de una cuenta combinado con la valoración que hacen sus seguidores de ese mismo esfuerzo. Como tal, el IGPP tiene en cuenta el esfuerzo de una cuenta en la doble faceta comunicadora que requiere la interactividad del proceso en Twitter: la conversadora, para la que el IGPP atiende al grado de feedback que proporciona la cuenta en su faceta receptor, y la emisora, que viene determinada por la valoración que de los mensajes de la cuenta hacen aquellos que los leen (feedback de la audiencia). 
Así, de dos cuentas con un mismo coeficiente de comunicación, será potencialmente más persuasiva aquella, cuyos mensajes tengan un mayor alcance (mayor feedback de la audiencia); es decir, aquella cuyos seguidores contribuyan más a difundir los mensajes de la cuenta seguida. Y viceversa, de dos cuentas con un mismo coeficiente de penetración, diremos que es potencialmente más persuasiva aquella cuyos mensajes impliquen más conversación (la que proporciona un mayor feedback como seguidora); es decir, la que presente un mayor coeficiente de comunicación. La expresión matemática de este razonamiento es la siguiente: IGPP $=\mathrm{Ccm} \times$ Alc. Por tanto, un gran esfuerzo de comunicación (elevado $\mathrm{Ccm}$ ) de una cuenta no se traducirá en un potencial persuasivo elevado si no se ve confirmada por la experiencia de uso, concretamente, por un cierto refrendo de su audiencia y que mide el coeficiente de penetración o alcance. Asimismo, para que una cuenta cuyos mensajes obtienen un notable respaldo de sus seguidores $(\mathrm{Alc})$ pueda alcanzar un mayor IGPP, deberá incrementar su interactividad, medida esta con el coeficiente de comunicación.

Para la aplicación de las fórmulas de los coeficientes descritos se requiere la recuperación de una serie de datos (variables) del tráfico de mensajes emitidos por la cuenta cuyo IGPP se desee calcular. En el anexo, se describen tales variables y los métodos de obtención de cada una de ellas.

\section{Conclusión}

El IGPP es un indicador relativo del potencial de persuasión que desarrolla una cuenta en las redes sociales que se constituyen con Twitter como vehículo comunicacional. No ha sido concebido para cuantificar los efectos de la persuasión o el grado de influencia ejercido.

No obstante, su diseño sí tiene en cuenta el grado de aceptación que, en los receptores, pueda suponer la retransmisión por estos de mensajes (efecto retuiteo). Trata de medir la fuerza o el poder de comunicación de quien, con razones y mediante una interacción personal y directa, pretende inducir a sus seguidores a adoptar determinadas decisiones en asuntos sociales o de orden político. Por consiguiente, es de especial utilidad para líderes de opinión y nuevos prescriptores sociales en general, aunque tam- 
bién es susceptible de ser aplicado a cuentas institucionales de servicio público y a las de marketing comercial. El valor que alcanza el IGPP de una determinada cuenta adquiere significado cuando se le compara con el de otra/s cuenta/s. Para que la comparación sea válida, las mediciones deben ser homogéneas.

La homogeneidad se consigue relativizando la actividad con respecto al tiempo (coeficiente de actividad) y el potencial comunicador con respecto a la actividad relativa (coeficiente de comunicación). Finalmente, el grado de propagación o alcance potencial se basa en un valor histórico, como es el que determina el alcance, al tener en cuenta el número medio de transmisiones efectuadas por mensaje retransmitido. Por tanto, la medición del IGPP proporciona información sobre el grado de incidencia que tienen las distintas dimensiones de la comunicación en red en los potenciales efectos persuasivos que se pueden conseguir.

La factorización del IGPP permite identificar las facetas del comportamiento comunicacional (proactividad, utilidad de la información, interactividad, personalización del mensaje) que conviene que sean reforzadas para poder ser más persuasivo. En la comunicación de dirigentes políticos, aunque el IGPP relativiza el peso que tiene el número de seguidores de una cuenta en el potencial persuasivo, sí tiene en cuenta la reciprocidad en el seguimiento atribuible a un posible efecto de homofilia política.

$\mathrm{Al}$ asignar un peso comunicacional nulo a la comunicación unidireccional del dirigente, el IGPP soslaya en gran medida el sesgo propagandístico inherente a toda comunicación política. Aunque el método propuesto ha sido diseñado con el propósito de evaluar el esfuerzo de comunicación de líderes de opinión y dirigentes políticos, resulta aplicable a cualquier colectivo, cuyos miembros tengan un perfil profesional común. Así permite medir el potencial persuasivo en Twitter de periodistas, community managers, jefes de prensa o agentes de marketing. 


\section{Referencias}

Abejón, P., Sastre, A. y Linares, V. (2012). Facebook y Twitter en campañas electorales en España. Anuario Electrónico de Estudios en Comunicación Social Disertaciones, 5(1), 129-159. Recuperado de http:// erevistas.saber.ula.ve/index.php/Disertaciones/

Aharony, N. (2012). Twitter use by three political leaders: an exploratory analysis. Online Information Review, 36(4), 587-603.

Álvarez, D. y Rodríguez, R. (2014). Parlamentarios 2.0: presencia y actividad de diputados y senadores españoles en las redes sociales. En R. Rubio (coord.), Parlamentos abiertos: tecnología y redes para la democracia (pp. 235-276). Madrid: Congreso de los Diputados.

Anger, I. y Kittl, C. (2011, septiembre). Measuring influence on Twitter. En Proceedings of the 11th International Conference on Knowledge Management and Knowledge Technologies (p. 31). ACM.

Aral, S. y Walker, D. (2012). Identifying influential and susceptible members of social networks. Science, 337(6092), 337-341.

Arriagada, A. y Schuster, M. (2008). Consumo de medios y participación ciudadana de los jóvenes chilenos. Cuadernos de Información, 22, 34-41.

Bakshy, E., Hofman, J. M., Mason, W. A. y Watts, D. J. (2011a, febrero). Everyone's an influencer: Quantifying influence on Twitter. En Proceedings of the fourth ACM International Conference on Web Search and Data Mining (pp.65-74). ACM.

Bakshy, E., Hofman, J. M., Mason, W. A. y Watts, D. J. (2011b, febrero). Identifying influencers on Twitter. En Fourth ACM International Conference on Web Seach and Data Mining (WSDM).

Bardin, L. (1996). Análisis de contenido. Madrid: Akal. 
Bennett, W. L. y Iyengar, S. (2008). A new era of minimal effects? The changing foundations of political communication. Journal of Communication, 58(4), 707-731.

Berelson, B. (1971). Content analysis in communication research. Nueva York: Hafner.

Borondo, J., Morales, A., Losada, J. y Benito, R. (2012). Characterizing and modeling an electoral campaign in the context of Twitter: 2011 spanish presidential election as a case study. Chaos: An Interdisciplinary Journal of Nonlinear Science, 22(2), 023138-023138-7.

Boyd, D., Golder, S. y Lotan, G. (2010, enero). Tweet, tweet, retweet: Conversational aspects of retweeting on Twitter. En System Sciences (HICSS), 2010 43rd Hawaii International Conference on (pp. 1-10). IEEE.

Castells, M. (2008). Comunicación y contrapoder en la sociedad red (I): los medios y la política. Telos: Cuadernos de Comunicación, Tecnología y Sociedad, 74, 12-24.

Cha, M., Haddadi, H., Benevenuto, F. y Gummadi, P. K. (2010). Measuring user influence in Twitter: the million follower fallacy. ICWSM, 10(10-17), 30.

Chaiken, S. y Maheswaran, D. (1994). Heuristic processing can bias systematic processing: effects of source credibility, argument ambiguity, and task importance on attitude judgment. Journal of Personality and Social Psychology, 66(3), 460.

Chaiken, S., Wood, W. y Eagly, A. H. (1996). Principles of persuasion. En A. W. Kruglanski y E. T. Higgins (eds.), Social psychology: Handbook of basic principles (pp.702-742). Nueva York: Guilford Press. 
Chang, H. (2010). A new perspective on Twitter hashtag use: Diffusion of innovation theory. Proceedings of the American Society for Information Science and Technology, 47(1), 1-4.

Gaffney, D. (2010). \# iranElection: Quantifying online activism. Recuperado de http://journal.webscience.org/295/2/websci10_submission_6.pdf

García, A., García, I. y Varona, D. (2012). Incidencia de las redes sociales vs cibermedios en las elecciones en España 2011. Enl@ce: Revista Venezolana de Información, Tecnología y Conocimiento, 9(2), 11-29.

Golbeck, J., Grimes, J. M. y Rogers, A. (2010). Twitter use by the US Congress. Journal of the American Society for Information Science and Technology, 61(8), 1612-1621.

Grant, W. J., Moon, B. y Busby, J. (2010). Digital dialogue? Australian politicians' use of the social network tool Twitter. Australian Journal of Political Science, 45(4), 579604.

Hanneman, R. (2001). Introducción a los métodos del análisis de redes sociales. Riverside: Universidad de California.

Heider, F. (1982). The psychology of interpersonal relations. New Jersey: Lawrence Erlbaum.

Hopcroft, J., Lou, T. y Tang, J. (2011, octubre). Who will follow you back?: Reciprocal relationship prediction. En Proceedings of the 20th ACM International Conference on Information and Knowledge Management (pp. 1137-1146). ACM.

Howard, P. N., Duffy, A., Freelon, D., Hussain, M. M., Mari, W. y Mazaid, M. (2011). Opening closed regimes: What was the role of social media during the Arab Spring? Available at SSRN 2595096. 
Izquierdo, L. (2012). Las redes sociales en la política española: Twitter en las elecciones de 2011. Estudos em Comunicação, 11, 149-164.

Jackson, N. y Lilleker, D. (2011). Microblogging, constituency service and impression management: UKMPs and the use of Twitter. The Journal of Legislative Studies, 17(1), 86-105.

Johnson-Cartee, K. S. y Copeland, G. A. (2003). Strategic political communication: rethinking social influence, persuasion, and propaganda. USA Lanham, Maryland: Rowman y Littlefield Publishers.

Katz, E.y Lazarsfeld, P. F. (1955). Personal influence: The part played by people in the flow of mass communications. Illinois: Free Press.

Khanafiah, D. y Situngkir, H. (2004). Social balance theory: Revisiting heider's balance theory for many agents. Recuperado de http:// arxiv.org/ftp/nlin/papers/0405/0405041.pdf

Khondker, H. H. (2011). Role of the new media in the Arab Spring. Globalizations, 8(5), 675-679.

Krippendorff, K. (1989). Content analysis. En E. Barnouw, G. Gerbner, W. Schramm, L. Worth y L. Gross (eds.), International encyclopedia of communication (pp. 403-407, vol. 1). Nueva York: Oxford University Press.

Krippendorff, K. y Wolfson, L. (1990). Metodología de análisis de contenido: teoría y práctica. Barcelona: Paidós.

Kwak, H., Lee, C., Park, H. y Moon, S. (2010, abril). What is Twitter, a social network or a news media? En Proceedings of the 19th International Conference on World Wide Web (pp. 591-600). ACM.

Larsson, A. O. y Moe, H. (2012). Studying political microblogging: Twitter users in the 2010 swedish election campaign. New Media y Society, 14(5), 729-747. 
Lassen, D. S. y Brown, A. R. (2011). Twitter the electoral connection? Social Science Computer Review, 29(4), 419-436.

Lotan, G., Graeff, E., Ananny, M., Gaffney, D., Pearce, I. y Boyd, D. (2011). The revolutions were tweeted: Information flows during the 2011 tunisian and egyptian revolutions. International Journal of Communication, 5, 1375-1405.

Mancera, A. y Pano, A. (2013). El discurso político en Twitter. Barcelona: Anthropos.

McNair, B. (2011). An introduction to political communication. Nueva York: Routledge.

Merton, R. K. (1948). Patterns of influence: A study of interpersonal influence and of communications behavior in a local community. Communications Research, 1949, 180-219.

Molina, J. L. (2001). El análisis de redes sociales. Barcelona: Bellaterra.

Moya, M. (2015). Análisis comunicacional del uso que los diputados españoles hacen de Twitter: evolución e implicaciones prácticas (Tesis doctoral, Universidad Carlos III, Madrid, España).

Park, C. S. (2013). Does Twitter motivate involvement in politics? Tweeting, opinion leadership, and political engagement. Computers in Human Behavior, 29(4), 1641-1648.

Parmelee, J. H. y Bichard, S. L. (2011). Politics and the twitter revolution: How tweets influence the relationship between political leaders and the public. Plymouth: Lexington Books.

Requena, F. (2003). Análisis de redes sociales (orígenes, teorías y aplicaciones). Madrid: Centro de Investigaciones Sociológicas.

Rogers, E. (1983). Diffusion of innovations. Nueva York: The Free Press. 
Scherman, A. y Arriagada, A. (2010, abril). ¿Ciudadanía digital, participación tradicional? Jóvenes, participación política y consumo de medios en Chile (pp. 15-17). En 3. ${ }^{\text {er }}$ Congreso Latinoamericano de Opinión Pública. Querétaro, México.

Scherman, A., Arriagada, A. y Valenzuela, S. (2013). La protesta en la era de las redes sociales: el caso chileno. En A. Arriagada y P. Navia, Intermedios: medios de comunicación y democracia en Chile (pp. 179197). Santiago: Ediciones Universidad Diego Portales.

Schramm, W. (1954). How communication works. En W. Schramm (ed.), The process and effects of communication. Urbana: University of Illinois Press.

Schwarz, N., Bless, H. y Bohner, G. (1991). Mood and persuasion: Affective states influence the processing of persuasive communications. Advances in Experimental Social Psychology, 24, 161-199.

Segado-Boj, F., Díaz-Campo, J. y Lloves-Sobrado, B. (2015). Líderes latinoamericanos en Twitter: viejas costumbres para nuevos medios en tiempos de crisis políticas. Revista Latina de Comunicación Social, 70, 156-173.

Stieglitz, S., Brockmann, T. y Xuan, L. D. (2012). Usage of social media for political communication. En 16th Pacific Asia Conference on Information Systems (PACIS), Paper 341.

Stieglitz, S. y Dang-Xuan, L. (2012, enero). Political communication and influence through microblogging--An empirical analysis of sentiment in Twitter messages and retweet behavior. En System Science (HICSS), 2012 45th Hawaii International Conference on (pp. 3500-3509). IEEE.

Taylor, H. F. (1967). Balance and change in the two-person group. Sociometry, 30, 262-279. 
Theocharis, Y., Lowe, W., Van Deth, J. W. y García Albacete, G. M. (2013). Using Twitter to mobilise protest action: Transnational online mobilisation patterns and action repertoires in the Occupy Wall Street, Indignados and Aganaktismenoi Movements (February 18, 2013).

Tremayne, M. (2014). Anatomy of protest in the digital era: A network analysis of Twitter and Occupy Wall Street. Social Movement Studies, 13(1), 110-126.

Trusov, M., Bodapati, A. V. y Bucklin, R. E. (2010). Determining influential users in internet social networks. Journal of Marketing Research, 47(4), 643-658.

Tumasjan, A., Sprenger, T. O., Sandner, P. G. y Welpe, I. M. (2010). Predicting elections with Twitter: What 140 characters reveal about political sentiment. ICWSM, 10, 178-185.

Weng, J., Lim, E. P., Jiang, J. y He, Q. (2010, February). Twitterrank: Finding topic-sensitive influential twitterers. En Proceedings of the Third ACM International Conference On Web Search And Data Mining (pp. 261-270). ACM.

Yanes, R. (2007). La comunicación política y los nuevos medios de comunicación personalizada. Ámbitos: Revista Internacional de Comunicación, 16, 355-365.

Zepeda, A. V., Franco, D. A. H. y Aldrete, A. A. (2011). Las cibercampañas en América Latina: potencialidades y limitantes. Correspondencias y Análisis, 1, 3-16.

Zhou, Z., Bandari, R., Kong, J., Qian, H. y Roychowdhury, V. (2010, July). Information resonance on Twitter: Watching Iran. En Proceedings of the First Workshop on Social Media Analytics (pp. 123-131). ACM. 


\section{Anexo}

Para el cálculo del IGPP, son necesarias las variables contenidas en la tabla 1.

\section{Tabla 1. Lista de variables y métodos de obtención}

\begin{tabular}{|c|c|c|}
\hline Variable & Descripción & Obtención \\
\hline Alta & Fecha de alta en Twitter & Twitonomy \\
\hline Fecha & Día y hora en las que se emitió el mensaje & Twitonomy \\
\hline Texto & Texto del mensaje & Twitonomy \\
\hline Per & Número de días del periodo de análisis & Calculada \\
\hline Msg & Número de mensajes emitidos (tuitetos, retuiteos y respuestas) & Twitonomy \\
\hline Rtn & Número de retuiteos nativos & Twitonomy \\
\hline $\mathrm{Me}$ & Número de menciones emitidas por la cuenta & Twitonomy \\
\hline $\mathrm{Rp}$ & Número de respuestas emitidas por la cuenta & Twitonomy \\
\hline $\mathrm{Tb}$ & Número tuiteos generados por la cuenta & Calculada \\
\hline ncRt & Número de cuentas únicas de las que esta ha retuiteado los Rtn mensajes & Calculada \\
\hline ncRp & Número de cuentas únicas a las que esta ha enviado mensajes de respuesta & Calculada \\
\hline txMsg & Número de veces que han sido retransmitidos los Msg emitidos por esta cuenta & Twitonomy \\
\hline txRT & $\begin{array}{l}\text { Número de veces que han sido retuiteados los mensajes no generados pero emitidos por } \\
\text { esta cuenta }\end{array}$ & Calculada \\
\hline txTx & Número de veces que han sido retuiteados los mensajes generados por la cuenta & Calculada \\
\hline Rtt & Número de mensajes emitidos por la cuenta que han sido retuiteados & Calculada \\
\hline Rtp & Número de mensajes generados por la cuenta que han sido retuiteados & Calculada \\
\hline Tseg & Número total de seguidores & Friendorfollow \\
\hline Seg-u & Número de seguidores únicos & Friendorfollow \\
\hline Seg-r & Número de seguidores recíprocos & Friendorfollow \\
\hline Seg-dos & Número de cuentas seguidas & Friendorfollow \\
\hline
\end{tabular}

Twitonomy ${ }^{8}$ F Friendorfollow 9 son las dos herramientas en línea con las que hemos recuperado parte de las variables necesarias para calcular los diversos coeficientes (de actividad, de comunicación y alcance) que forman parte del IGPP. Las restantes variables han sido calculadas a partir de los datos recuperados, así:

$$
\begin{aligned}
& \text { - } \quad \text { Per=Alta-Fecha } \\
& \text { - } \quad \mathrm{Tb}=\text { Msg-(Rtn-Rp) }
\end{aligned}
$$

8 https://www.twitonomy.com/

9 https://friendorfollow.com/ 
- ncRt: valor obtenido contabilizando el número de cuentas únicas a las que se han retuiteado sus Rtn mensajes

- ncRp: valor obtenido contabilizando el número de cuentas únicas a las que se han enviado Rp mensajes de respuesta

- txRT: valor obtenido contabilizando el número de veces que han sido retuiteados los mensajes emitidos por una cuenta pero generados por otra

- $\quad$ txTx=txMsg-txRt

- Rtt: valor obtenido contabilizando el número de mensajes emitidos por una cuenta que han sido retuiteados

- $\quad$ Rtp: valor obtenido contabilizando el número de mensajes generados por la cuenta que han sido retuiteados 